\title{
Detección de isoenzimas sintasas de óxido nítrico (NOS) en membrana sinovial de articulaciones metacarpofalángicas equinas normales y con daño macroscópico ${ }^{\#}$
}

\author{
Detection of nitric oxide synthase isoenzymes (NOS) in normal and alterated synovial membrane \\ from equine metacarpophalangeal joint
}

\author{
M Galleguillos ${ }^{a^{*},}$ F Núñez ${ }^{a}$, ME Letelier ${ }^{b}$, B Contesse $^{\mathrm{a}}$, H Adarmes $^{\mathrm{a}}$

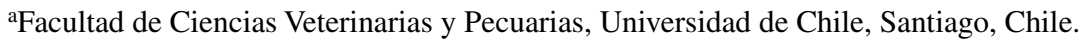 \\ bFacultad de Ciencias Químicas y Farmacéuticas, Universidad de Chile, Santiago, Chile.
}

\begin{abstract}
SUMMARY
We studied the presence of NOS isoforms in synovial membrane from equine metacarpophalangeal joint. This was considered a normal joint group $\mathrm{NN}(\mathrm{n}=13)$ when there was not any visual damage of joint structures. The damaged groups were classified as follows: NA $(n=8)$ with congestive synovial membrane without cartilage damage; AN $(\mathrm{n}=10)$ cartilage damage and normal synovial membrane and AA $(\mathrm{n}=8)$ those with cartilage damage and congestive synovial membrane. Damaged cartilage showed erosions and multiple wear lines. Detection of NOS isoforms was performed by western blot using monoclonal antibodies. The generated chemiluminescence was measured and expressed in densitometric relative units (DRU) that represent the rate between the pixels of each sample and those of the standard. NOS I showed a $145 \mathrm{kDa}$ band in all samples, without significant differences between joint groups. NOS III showed a $140 \mathrm{kDa}$ band, with detection percentages of NN $=77 \% ; \mathrm{NA}=63 \% ; \mathrm{AN}=30 \%$ and $\mathrm{AA}=100 \%$, without significant differences between them. NOS II was no detected in these samples. In conclusion, NOS I and partially NOS III, were expressed in a constitutive form in equine synovial membrane, while NOS II was not detected in these assays, which was likely to be expected given the absence of acute inflammation, at least during macroscopic observation.
\end{abstract}

Palabras clave: membrana sinovial, isoformas NOS, enfermedad articular.

Key words: synovial membrane, NOS isoforms, joint disease.

\section{INTRODUCCIÓN}

La osteoartritis $(\mathrm{OA})$ afecta las articulaciones sinoviales y se caracteriza por daño del cartílago articular, esclerosis del hueso subcondral, formación de osteofitos marginales y alteraciones de los tejidos blandos (McIlwraith, 1996, Cantley y col 1999). Una de las causas de esta patología es el trauma, que puede ser único y de alta intensidad, o bien de menor intensidad, pero repetitivo en el tiempo (Cantley y col 1999) y particularmente en condiciones de fuerzas biomecánicas inapropiadas (Goodrich y Nixon 2006). La consecuencia es un cuadro inflamatorio inicial que se presenta en forma de sinovitis y capsulitis (McIlwraith 1996), que se caracteriza por infiltración de neutrófilos y macrófagos en el tejido articular (Goldring 2000), y por el aumento de citoquinas proinflamatorias como son la interleuquina $1-\beta$ (IL-1 $\beta$ ) y el factor de necrosis tumoral- $\alpha$ (TNF- $\alpha)$. Se ha establecido que la IL- $1 \beta$ juega un papel central en la fisiopatología de la OA (Dinarello 1988) ya que induce en el condrocito una cascada de procesos catabólicos que incluye la expresión génica de

Aceptado: 06.10.2010.

\# Proyecto DID I-14-2/2001, Universidad de Chile.

* Casilla 2 correo 15, La Granja, Santiago, Chile; mgallegu@ uchile.cl metaloproteinasas de matriz, de la sintasa inducible de óxido nítrico (NOS II), ciclooxigenasa-2 y de la sintasa-1 microsomal de prostaglandina $\mathrm{E}_{2}\left(\mathrm{mPGE}_{2}\right)$ (Stadler y col 1991, Morisset y col 1998).

El NO es un radical libre gaseoso sintetizado a partir de la oxidación del aminoácido L-arginina, reacción catalizada por la sintasa del óxido nítrico (NOS). Se han descrito principalmente tres isoenzimas para la NOS: NOS I o neuronal (nNOS), NOS II o inducible (iNOS) y NOS III o endotelial (eNOS) (Clancy y col 1998, Lee y col 2002). La NOS-I y la NOS-III son descritas como constitutivas (cNOS), sin embargo, pueden sufrir regulación génica frente a distintos estímulos como la hipoxia, el ejercicio, hipertensión pulmonar entre otros (Förstermann y col 1998). La NOS II es de tipo inducible e independiente de la presencia $\mathrm{Ca}^{+2}$, lo que hace que esta enzima pueda generar una mayor cantidad de NO que las isoformas NOS-I y NOS-III (Tomita y col 2001).

Los condrocitos constituyen la fuente más importante de NO a nivel articular (Jang y Murrel 1998). Se ha descrito un aumento de $\mathrm{NO}$ en el cartílago osteoartrítico equino (van der Harst y col 2006), así como en las capas superficiales del cartílago normal humano frente al estímulo con citocinas proinflamatorias (Hayashi y col 1997). En la membrana sinovial de articulaciones humanas se ha descrito, a través del análisis inmunohistoquímico, que la NOS II se expresa 
intensamente en cuadros inflamatorios tanto agudos como crónicos, y no así en articulaciones normales (Di Mauro y col 2006). Por lo tanto, se plantea como objetivo de este trabajo evaluar la presencia del conjunto de isoformas de NOS en la membrana sinovial del equino en diferentes condiciones articulares.

\section{MATERIAL Y MÉTODOS}

\section{MATERIAL BIOLÓGICO}

Se utilizaron articulaciones metacarpofalángicas de equinos de matadero. Mediante inspección visual post mortem se seleccionaron articulaciones normales y otras con alteración del cartílago y/o de la membrana sinovial. Cada articulación seleccionada provino de equinos diferentes. Se consideraron normales (NN) aquellas articulaciones $(n=13)$ con un cartílago de superficie lisa y brillante, de color blanco nacarado y una membrana sinovial sin signos de congestión. Las articulaciones alteradas fueron divididas en aquellas que presentaron únicamente congestión de la membrana sinovial (NA) $(n=8)$; aquellas que presentaron únicamente daño del cartílago articular como focos de erosión y líneas de roce $(\mathrm{AN})(\mathrm{n}=10)$ y aquellas que presentaron tanto daño del cartílago como congestión de la membrana sinovial (AA) $(n=8)$. En todos los casos se consideró para la selección que el líquido sinovial (LS) debía presentar un color amarillo claro, translúcido y sin derrame sanguinolento, de lo contrario la articulación sería eliminada (Adarmes y col 2009). Las muestras de membrana sinovial se obtuvieron desde la zona palmar de la articulación, a nivel de los sacos volares. Luego se etiquetaron y conservaron $\mathrm{a}-20^{\circ} \mathrm{C}$ hasta su procesamiento.

\section{PREPARACIÓN DE LOS HOMOGENEIZADOS DE MEMBRANA} SINOVIAL

A trozos entre 0,5 a $1 \mathrm{~g}$ de membrana sinovial se les adicionó en una proporción 1:4 una solución amortiguadora de Tris-HCl 50 mM, pH 7,4 y que contenía EDTA 0,1 mM, EGTA 0,1 mM, ditiotritol (DTT) 0,5 mM, inhibidores de proteasas (leupeptin $2 \mathrm{mM}$, pepstatin $1 \mathrm{mM}$, fluoruro de fenilmetilsulfonilo (PMSF) $1 \mathrm{mM}$ y 2 - $\beta$-mercaptoetanol $12 \mathrm{mM}$. Esta mezcla se homogeneizó en un ultraturrax a $13.000 \mathrm{rpm}$ por tres veces durante 15 segundos. Luego este homogeneizado se centrifugó a 1.000 x $g$ por 20 minutos y se obtuvo el sobrenadante que se conservó a $-20{ }^{\circ} \mathrm{C}$.

\section{DETERMINACIÓN DE PROTEÍNAS}

Se utilizó el método espectrofotométrico de Lowry y col (1951). Se usó seroalbúmina de bovino como estándar. La concentración de proteínas de cada homogeneizado permitió que los ensayos de determinación de las distintas isoformas de NOS fueran comparables.
DETECCIÓN DE LAS ISOFORMAS DE NOS EN MEMBRANA SINOVIAL

Cada homogeneizado se diluyó en una relación 1:3 en una solución amortiguadora de Tris- $\mathrm{HCl} 33 \mathrm{mM}$ pH 6,8 que contenía SDS $10 \%$, glicerol 1\%, azul de bromofenol $0,005 \%$ y $\beta$-mercaptoetanol $700 \mathrm{mM}$. Para la electroforesis se utilizó poliacrilamida al $8 \%$ en el gel separador y al $5 \%$ en el gel separador, con un voltaje constante de 150 volts durante 80 minutos. En el gel se cargaron $50 \mu \mathrm{g}$ de proteína de cada muestra. Los geles se transfirieron a membranas PVDF (Immun-Blot-Bio-Rad) por medio de electrotransferencia a 0,35 A constante por dos horas. Las membranas se bloquearon toda la noche a $4{ }^{\circ} \mathrm{C}$ en una solución de TBS-Leche-Tween-20 (Tris- $\mathrm{HCl}$ $20 \mathrm{mM}$ pH 7,6, NaCl $137 \mathrm{mM}$, leche descremada 6\% y Tween-20 0,05\%). Luego se realizaron tres lavados de 10 minutos cada uno, con agitación constante en una solución de TBS-Tween 20 (Tris-HCl 20 mM pH 7,6, $\mathrm{NaCl} 137$ mM y Tween-20 0,05\%) y después las membranas se incubaron durante dos horas y con agitación en una solución de TBS-Leche-Tween-20 en presencia de los anticuerpos monoclonales anti-nNOS (humano, Catálogo N31020-050, Transduction Laboratories), anti-iNOS (ratón, Catálogo N39120-050, Transduction Laboratories) y anti-eNOS (humano, Catálogo N30020050, Transduction Laboratories). Se utilizaron diluciones 1:500 para los anticuerpos anti-iNOS y anti-eNOS y de 1:4000 para el anticuerpo anti-iNOS. Se realizó un lavado similar a los anteriores y luego las membranas se incubaron, por una hora con agitación, en presencia de un segundo anticuerpo anti-IgG de ratón (anticuerpo ImmunePure, Pierce) conjugado con peroxidasa de rabano en solución TBS-Leche-Tween-20. Posteriormente se agregó un sustrato (SuperSignal, Pierce) generador de un producto quimioluminiscente por acción de la peroxidasa. La reacción se evidenció por bandas luminosas, las que se expusieron a películas fotosensibles (CL-X Posure, Pierce) para registrarlas. Luego, cada film se escaneó y se sometió a un análisis densitométrico usando el programa computacional INI-SCAN-IT gel versión 4.1, que permitió la cuantificación de pixeles de cada banda. Los resultados se expresaron en unidades densitométricas relativas, que correspondieron a la razón entre los pixeles de cada banda y los pixeles de un patrón interno.

\section{ANÁLISIS ESTADÍSTICO}

Las unidades relativas de cada grupo se expresan como promedio \pm desviación estándar, las cuales se sometieron a un análisis estadístico mediante la prueba de ANOVA para establecer las diferencias entre la cantidad de isoenzima detectada en las diferentes alteraciones articulares (Infostat 2004). 


\section{RESULTADOS Y DISCUSIÓN}

Se detectó la NOS I en el ciento por ciento de las muestras, sin variaciones significativas en su cantidad relativa entre los grupos analizados, lo que indicaría que su expresión es constitutiva en la membrana sinovial sin que exista relación con la condición articular. El cuadro 1 muestra el análisis densitométrico expresado en unidades densitométricas relativas (UDR) de NOS I y NOS III en los distintos grupos articulares. La NOS I que se ha encontrado expresada en sistema nervioso central y periférico de diferentes mamíferos, también se ha detectado en otros tejidos y células no neuronales de varias especies animales (Förstermann y col 1998).

Cuadro 1. Análisis densitométrico de NOS I y III en membrana sinovial de la articulación metacarpofalángica equina en diferentes condiciones articulares.

Densitometric analysis of synovial membrane NOS I and III in equine metacarpophalangeal joint with different joint condition

\begin{tabular}{cccccc}
\hline $\begin{array}{c}\text { Grupo } \\
\text { Articular }\end{array}$ & $\mathrm{n}$ & $\begin{array}{c}\text { NOS I } \\
\text { UDR } \pm \mathrm{DE}\end{array}$ & $\mathrm{n}$ & $\begin{array}{c}\text { NOS III } \\
\text { UDR } \pm \mathrm{DE}\end{array}$ & $\mathrm{n}$ \\
\hline NN & 13 & $0,84 \pm 0,64$ & 13 & $0,125 \pm 0,98$ & 10 \\
NA & 8 & $0,58 \pm 0,34$ & 8 & $0,143 \pm 0,083$ & 5 \\
AN & 10 & $0,71 \pm 0,82$ & 10 & $0,295 \pm 0,193$ & 3 \\
AA & 8 & $0,63 \pm 0,44$ & 8 & $0,134 \pm 0,129$ & 8 \\
\hline
\end{tabular}

n: $\quad$ Número de muestras

UDR: Unidades densitométricas relativas

NN: Cartílago y membrana sinovial macroscópicamente normales

NA: Cartílago normal y membrana sinovial congestiva

AN: Cartílago alterado y membrana sinovial normal

AA: Cartílago alterado y membrana sinovial congestiva

En la figura 1 se demuestra a través de western blot que la masa molecular de la NOS I de membrana sinovial equina corresponde a $145 \mathrm{kDa}$, para todas las muestras analizadas, mientras que el control positivo suministrado por el fabricante (NOS I de tejido nervioso humano) es de $155 \mathrm{kDa}$ que representa la forma descrita en la literatura para tejido nervioso. Otro control positivo utilizado, un homogeneizado de corteza cerebral equina, mostró (cer) también una masa molecular de $155 \mathrm{kDa}$ (figura 1). Un resultado semejante fue obtenidos por Amin y col (1995), que determinó la presencia de una isoforma de NOS I de $150 \mathrm{kDa}$ en condrocitos obtenidos de pacientes humanos con osteoartritis, que corresponde a una masa molecular menor a la que se encuentra habitualmente en sistema nervioso (155 a $160 \mathrm{kDa})$. Una explicación de este resultado se relacionaría con la compleja organización genómica de NOS I, que daría origen a diferentes transcriptos de ARNm (Förstermann y col 1998), lo que hace posible generar variantes de esta enzima en una expresión de tipo tejido-específica. Por ejemplo, en humanos se ha determinado que por uso de promotores alternativos existe una isoforma de NOS I expresada en testículo, denominada TnNOS, que se expresa específicamente en las células de Leydig y tiene una masa molecular $125 \mathrm{kDa}$. Esta variante, a diferencia de la NOS I encontrada en tejido cerebral, carece del motivo de interacción proteína-proteína PDZ/ GLGF, el cual determina su localización subcelular en la membrana plasmática (Wang y col 2002).

En relación a la expresión de la NOS III, sólo se detectó la enzima en el 67\% del total de articulaciones analizadas (cuadro 1). Una posible explicación a este hecho es que en aquellas muestras en las cuales no se detectó NOS III existiría una baja expresión de la enzima, por debajo de los límites de detección de la técnica utilizada. Otra posibilidad es que la muestra de tejido extraída en algunos casos haya contenido un bajo contenido de endotelio vascular, tejido rico en NOS III. En cuanto a la masa molecular determinada para NOS III mediante "western blot" fue de $140 \mathrm{kDa}$ similar a la encontrada para los homogeneizados tanto de aorta humana como de equino (figura 2).
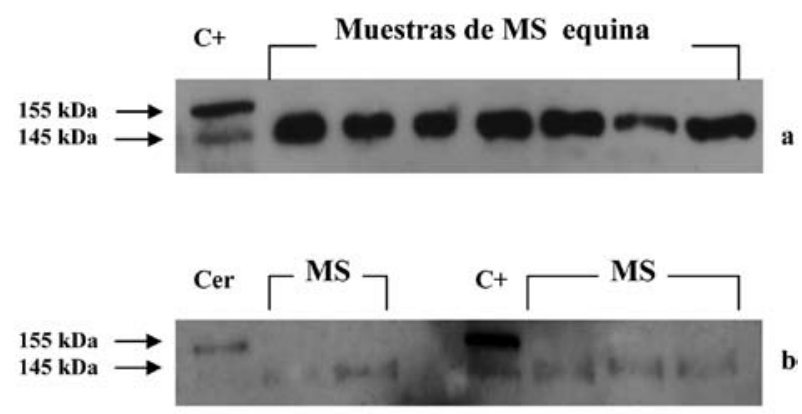

Figura 1. Análisis western blot de la NOS I. En (a) se muestra el control positivo $(\mathrm{C}+)$ para NOS I $(155 \mathrm{kDa})$ y los homogeneizados de MS (50 $\mu \mathrm{g}$ de proteína total), cuya isoenzima muestra una MM de $145 \mathrm{kDa}$. En (b) se muestra la isoenzima de un homogeneizado de corteza cerebral equina (cer) y el C+ con una MM de $155 \mathrm{kDa}$, y homogeneizados de MS equina con una MM de $145 \mathrm{kDa}$.

Western blot analysis of NOS I. Image (a) shows the positive $(\mathrm{C}+)$ control for NOS I (155 kDa) and the homogenized SM (50 $\mu \mathrm{g}$ of total protein) whose isoenzyme shows a MM of $145 \mathrm{kDa}$. Image (b) shows the isoenzyme of equine brain cortex homogenized (cer) and the $\mathrm{C}+$ with a MM of $155 \mathrm{kDa}$, and homogenized of SM equine with a MM of $145 \mathrm{kDa}$.

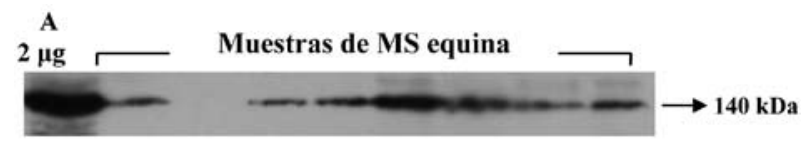

Figura 2. Análisis western blot de la NOS III. Se utilizaron $100 \mu \mathrm{g}$ de proteína total de MS. La muestra A corresponde al homogeneizado de endotelio de la arteria aorta equina $(2 \mu \mathrm{g})$ con una MM de $140 \mathrm{kDa}$ al igual que los homogeneizados de MS.

Western blot analysis of NOS III. $100 \mu \mathrm{g}$ of total protein of MS were analized with monoclonal antibodies anti-eNOS (1:500). The A sample is an endothelium homogenized of equine aorta artery $(2 \mu \mathrm{g})$ with a $140 \mathrm{kDa} \mathrm{MM}$ as well as the SM homogenized. 
No se detectó la presencia de la NOS II en ninguna de las muestras, a pesar de incrementar la concentración de proteína (hasta $100 \mu \mathrm{g} /$ pocillo), la dilución del anticuerpo anti-NOS II (1:500) y del tiempo de incubación (16 horas). El control positivo del fabricante, que consistió en un lisado de macrófagos de ratón activados por INF- $\gamma$ y LPS, sí fue detectado en los diferentes ensayos (dato no mostrado). Se ha descrito una homología del $90 \%$ entre los genes de las tres isoformas de NOS de diferentes especies de mamíferos (Nathan y Xie 1994).

En otro estudio, Di Mauro y col (2006) mediante inmunohistoquímica detectaron la presencia de NOS II en el tejido sinovial humano proveniente de rodilla con OA idiopática y en pacientes con inflamación postraumática. Por otra parte, los mismos autores no detectaron esta isoforma en el tejido sinovial normal. En la misma línea, Stefanovic-Racic y col (1994) describieron que sinoviocitos B de conejo cultivados en presencia de citocinas proinflamatorias (IL-1 $\beta$ y TNF- $\alpha$ ) produjeron cantidades significativas de nitrito, producto estable del $\mathrm{NO}$, lo que sugirió la inducción de la NOS II en esas condiciones, tal como ocurre en el macrófago por acción de estas mismas citoquinas y otros estímulos como LPS. También McInnes y col (1996) detectaron ARNm de NOS II en membrana sinovial de articulaciones osteoartríticas y con artritis reumatoidea en pacientes humanos, sin que aparecieran diferencias entre ellas. En cultivos de sinoviocitos y condrocitos de equino estimulados con IL- $1 \beta$ y con lipopolisacáridos (LPS), Frean y col (1997) demostraron que los sinoviocitos produjeron menores cantidades de nitrito con respecto a los condrocitos. Además, von Rechenberg y col (2000) demostraron en explantes de cartílago y de membrana sinovial de equino que la concentración de nitrito era mayor en el caso del cartílago. Simmons y col (1999) mostraron en membrana sinovial sana de equino una muy baja actividad NOS y no fue afectada por la exposición a IL-1 $\beta$ al menos seis horas después.

Estos antecedentes que muestran una relación entre inflamación y la presencia de NOS II sugieren que nuestros resultados representan condiciones articulares posiblemente de tipo no inflamatorio. Esto a pesar de que aparece una congestión moderada de la membrana sinovial tanto en el grupo con cartílago normal (NA) como en el grupo con cartílago dañado (AA); en ningún caso se detectó presencia de NOS II cuya expresión se induce por acción de mediadores proinflamatorios. Se debe considerar además que se descartaron aquellas muestras que presentaron un líquido sinovial sanguinolento o una elevada concentración de proteínas totales.

En el trabajo de Di Mauro y col (2006) se demostró también la expresión de NOS I y III en tejido sinovial humano tanto en los casos de OA como de inflamación postraumática. En el tejido normal también se observó expresión de estas isoformas, aunque en menor intensidad. Este resultado obtenido en humanos coincide parcialmente con nuestro estudio en articulación de equino, ya que demostramos la presencia de NOS I y III sin variaciones significativas entre los grupos articulares. Esto implicaría que su expresión es constitutiva en el tejido sinovial y que este tejido no sería la principal fuente de NO a nivel articular. Además, en un estudio anterior en que se utilizaron grupos articulares de equino similares a los de este trabajo (Adarmes y col 2009), se encontró que la concentración de nitrito del líquido sinovial no mostró diferencias entre los grupos, lo que podría indicar que la síntesis de NO dependería fundamentalmente de los condrocitos y de la actividad de la NOS constitutivas de la membrana sinovial en condiciones no inflamatorias. Sería interesante a futuro poder caracterizar mejor la NOS I de $145 \mathrm{kDa}$ detectada en este estudio, puesto que pudiera ser una isoforma tejido específica de la membrana sinovial equina.

\section{RESUMEN}

Se determinó la presencia de isoformas de sintasa del óxido nítrico (NOS) en membrana sinovial de articulación metacarpofalángica equina. Se consideró un grupo normal NN $(n=13)$ sin daño observable en las estructuras articulares; grupo NA $(n=8)$ con la membrana sinovial congestiva; grupo $\mathrm{AN}(\mathrm{n}=10)$ con el cartílago dañado y membrana sinovial normal; AA $(n=8)$ con el cartílago y membrana sinovial alterados. La detección de las isoformas de NOS se realizó mediante "Western Blot" utilizando anticuerpos monoclonales. La quimioluminiscencia generada fue medida y expresada en unidades densitométricas relativas (UDR) que corresponde a la razón entre los pixeles de cada muestra y de un patrón interno. La NOS I de 145 kDa se encontró en todas las muestras analizadas. La NOS III de 140 kDa se detectó sólo en un porcentaje de las muestras $(\mathrm{NN}=77 \% ; \mathrm{NA}=63 \% ; \mathrm{AN}=30 \% ; \mathrm{AA}=100 \%) \mathrm{y}$ no se encontró una diferencia significativa entre los grupos. No se detectó la NOS II en ninguna de las muestras analizadas. Se concluye que la NOS I y NOS III son constitutivas de la membrana sinovial, mientras que la NOS II no se detecta, posiblemente debido a que no existe una inflamación aguda en los grupos analizados.

\section{REFERENCIAS}

Adarmes H, JP Solís, A Müller, A Croxatto, M Galleguillos. 2009. Determinación de nitrito como metabolito estable del óxido nítrico en el líquido sinovial de la articulación metacarpofalángica equina. Arch Med Vet 41, 257-261.

Amin AR, PE Di Cesare, P Vyas, M Attur, E Tzeng, TR Billiar, S Stuchin, A Abrason. 1995. The expression and regulation of nitric oxide synthase in human osteoarthritis affected chondrocytes: evidence for up-regulated neuronal nitric oxide synthase. $J$ Exp Med 182, 2097-2102.

Cantley CH, E Firth, J Delahunt, D Pfeiffer, K Thompson. 1999. Naturally occurring osteoarthritis in the metacarpophalangeal joints of wild horses. Equine Vet J 31, 73-81.

Clancy RM, AR Amin, SB Abramson. 1998. The role of nitric oxide in inflammation and immunity. Arthritis Rheum 2, 1141-1151.

Di Mauro D, L Bitto, L D'Andrea, A Favaloro, O Giaccobe, L Magaudda, G Rizzo, F Trimarchi. 2006. Behaviour of nitric oxide synthase isoforms in inflammatory human joint diseases: an immunohistochemical study. Ital J Anat Embryol 111, 111-123.

Dinarello CA. 1988. Interleukin-1. Ann N Y Acad Sci 546, 122-132.

Förstermann U, J Boissel, H Kleinert. 1998. Expressional control of the constituve isoforms of nitric oxide synthase (NOS I and NOS II). Faseb J 12, 773-790.

Frean SP, CE Bryant, I Froling, J Elliot, P Lees. 1997. Nitric oxide production by equine articular cells in vitro. Equine vet $J$ 29, 98-102. 
Goldring MB. 2000. Osteoarthritis and cartilage: the role of cytokines. Curr Rheumatol Rep 2, 459-465.

Goodrich LR, AJ Nixon. 2006. Medical treatment of osteoarthritis in the horse - a review. Vet $J 171,51-69$.

Hayashi T, E Abe, T Yamate, Y Taguchi, HE Jasin. 1997. Nitric oxide production by superficial and deep articular chondrocytes. Arth Rheum 40, 261-269.

Infostat. 2004. Manual del usuario. Grupo Infostat, FCA, Universidad Nacional de Córdoba. Editorial Brujas. Córdoba. Argentina.

Jang D, G Murrell. 1998. Nitric oxide in arthritis. Free Radic Biol Med 24, 1511-1519.

Lee MS, MCD Trindale, T Ikenoue, DJ Schurman, SB Goodman, RL Smith. 2002. Effects of shear stress on nitric oxide and matrix protein gene expression in human osteoarthritic chondrocytes in vitro. J Orthop Res 20, 556-561.

Lowry OH, NJ Rosebrough, AL Farr, RJ Randall. 1951. Protein measurement with the Folin's phenol reagent. J Biol Chem 193, 265-275.

McIlwraith CW. 1996. General pathobiology of the joint and response to injure. In: McIlwraith $\mathrm{CW}$, Trotter $\mathrm{GW}$ (eds). Joint disease in the horse. WB Saunders, Philadelphia, USA, Pp 40-70.

McInnes IB, B Leung, M Field, X Wei, F Huang, R Sturrock, A Kinninmonth, J Weidner, R Mumford, F Liew. 1996. Production of nitric oxide in the synovial membrane of rheumatoid and osteoarthritis patiens. J Exp Med 184, 1519-1524.

Morisset S, C Patry, M Lora, AJ de Brum-Fernández. 1998. Regulation of cyclooxygenase- 2 expression in bovine chondrocytes in culture by interleukin 1 alpha, tumor necrosis factor-alpha, glucocorticoids, and 17 beta-estradiol. J Rheumatol 25, 1146-1153.

Nathan C, Q Xie. 1994. Nitric oxide synthases: roles, tolls and controls. Cell 78, 915-918.
Simmons EJ, A Bertone, J Hardy, S Weisborde. 1999. Nitric oxide synthase activity in healthy and interleukin 1beta-exposed equine synovial membrane. Am J Vet Res 60, 714-716.

Stadler J, M Stefanovic-Racic, TR Billiar, RD Curran, LA McIntyre, HI Georgescu, RL Simmons, CH Evans. 1991. Articular chondrocytes synthesize nitric oxide in response to cytokines and lipopolysaccaride. J Immunol 147, 3915-3930.

Stefanovic-Racic M, J Stadler, HI Georgescu, C Evans. 1994. Nitric oxide synthesis and its regulation by rabbit synoviocytes. J Rheumatol 37, 1892-1898.

Tomita M, EF Sato, M Nishikawa, Y Yamano, M Inoue. 2001. Nitric oxide regulates mitochondrial respiration and functions of articular chondrocytes. Arthritis Rheum 44, 96-104.

Van der Harst M, S Bull, PA Brama, AB Barneveld, PR van Weeren, $\mathrm{C}$ van de Lest. 2006. Nitrite and nitrotyrosine concentrations in articular cartilage, subchondral bone, and trabecular bone of normal juvenile, normal adult, and osteoarthritic adult equine metacarpophalangeal joints. J Rheumatol 33, 1662-1667.

Von Rechenberg B, CW McIlwraith, MK Akens, DD Frisbie, C Leutenegger, JA Auer. 2000. Spontaneous production of nitric oxide (NO), prostaglandin (PGE2) and neutral metalloproteinases (MMPs) in media of explant cultures of equine synovial membrane and articular cartilage from normal and osteoarthritic joints. Equine Vet J 32, 140-150.

Wang Y, DC Newton, TL Miller, AM Teichert, MJ Phillips, MS Davidoff, PA Marsden. 2002. An alternative promoter of the human neuronal nitric oxide synthase gene is expressed specifically in Leydig cells. Am J Pathol 160, 369-380. 